\title{
Neumann problem on the semi-line for the Burgers equation
}

Silvana De Lillo ${ }^{1,2}$ and Matteo Sommacal ${ }^{3^{*}}$

* Correspondence: sommacal@ihes. $\mathrm{fr}$

${ }^{3}$ Institut des Hautes Etudes Scientifiques, 91440 Bures-surYvette, France

Full list of author information is available at the end of the article

\section{Abstract}

In this article, the Neumann problem on the semi-line for the Burgers equation is considered. The problem is reduced to a nonlinear integral equation in one independent variable, whose unique solution is proven to exist for small time. An explicit solution is discussed as well.

Keywords: Burgers equation, Neumann problem

\section{Introduction}

Initial/boundary value (IBV) problems for integrable nonlinear PDEs frequently appear in physical applications and have originated several important studies in the past few decades. Much interest has been devoted to IBV problems for nonlinear PDEs which are treatable by the inverse scattering transform method, such as the nonlinear Shrödinger equation (NLS), the Korteweg-de Vries equation (KdV), and the Sine-Gordon equation [1-8]. Other studies have been devoted to IBV problems for nonlinear PDEs which are $C$-integrable, namely, which are exactly linearizable via a change of variables: well-known examples in this class are the Burgers equation and the Eckhaus equation [9-15].

It is the aim of this article to analyze the Neumann problem for the Burgers equation:

$$
u_{t}=u_{x x}+2 u_{x} u, \quad u \equiv u(x, t),
$$

on the semi-infinite domain $x \in[0, \infty)$ characterized by the following set of initial and boundary data:

$$
\begin{aligned}
& u(x, 0)=u_{0}(x), \quad x \geq 0, \\
& u_{x}(0, t)=F(t), \quad t \geq 0,
\end{aligned}
$$

with

$$
F(0)=u_{0_{x}}(0)
$$

where $F(t)$ is a continuous, bounded function of its argument:

$$
F(t) \leq B, \quad B \in \mathbb{R},
$$


and the initial datum $u_{0}(x)$ is assumed to be integrable on the semi-line:

$$
\int_{0}^{\infty}\left|u_{0}(x)\right| \mathrm{d} x<\infty
$$

We point out that such problem was previously considered in [12], where it was shown to be equivalent to a nonlinear integro-differential equation (in one independent variable), which however cannot generally be solved. In this article, our analysis is based on the method developed in [10] for the solution of Dirichlet problem and on the use of the contraction-mapping technique, analogously to what was done for the Eckhaus equation in [15]. In particular, the main result of the present study is to prove the following.

Theorem 1 There exists a finite constant $\sigma \in \mathbb{R}, 0<\sigma<\infty$, such that the solution to the Neumann problem (2a-2e) for (1) exists and is unique for $0 \leq t<\sigma$.

Unlike the Neumann problem on the finite interval $(0,1)$, for which the existence of a solution in $L^{2}(0,1)$ was proven in [16] by means of the Galerkin method, the Neumann problem on the semi-line for the Burgers equation has not received much attention in the literature in the past. To the best of the authors' knowledge, Theorem 1 (as well as Lemma 1 in Section 3) are new.

In Section 2, we put problem $(1,2 \mathrm{a}-2 \mathrm{e})$ in a one-to-one correspondence with a Neumann problem for the heat equation, characterized by a boundary datum which is a nonlinear combination of the boundary data $\left\{u(x, 0), u_{x}(0, t)\right\}$ of the Burgers equation. We reduce such a problem to a nonlinear integral equation of Volterra type in one independent variable $(t)$. In Section 3, we prove the existence and uniqueness of the solution for small time. In Section 4, we discuss a special solution of the problem $(1,2 \mathrm{a}-2 \mathrm{e})$.

\section{Reduction to a nonlinear integral equation}

We begin our analysis by introducing the following ("generalized" Hopf-Cole) linearizing transformation $[11,12]$ :

$$
\begin{aligned}
& v(x, t)=C(t) u(x, t) \exp \left[\int_{0}^{x} \mathrm{~d} \tilde{x} u(\tilde{x}, t)\right], \\
& u(x, t)=\frac{v(x, t)}{C(t)+\int_{0}^{x} \mathrm{~d} \tilde{x} v(\tilde{x}, t)}
\end{aligned}
$$

with

$$
C(0)=1
$$

The above transformation maps the Burgers equation (1) into the linear heat equation

$$
v_{t}(x, t)=v_{x x}(x, t),
$$

with the "compatibility" condition for $C(t)$ given by

$$
\dot{C}(t)=C(t)\left[u_{x}(0, t)+u^{2}(0, t)\right]
$$

where (hereafter) the dot indicates differentiation with respect to time. Through transformation (3a-3c), from the Neumann IBV data for the $u(x, t),(2 \mathrm{a}-2 \mathrm{e})$, we obtain 
the IBV data for the $v(x, t)$ that characterize $(4)$ :

$$
\begin{aligned}
& v(x, 0)=u_{0}(x) \exp \left[\int_{0}^{x} \mathrm{~d} \tilde{x} u_{0}(\tilde{x})\right] \\
& v(0, t)=C(t) u(0, t) \\
& v_{x}(0, t)=C(t)\left[u_{x}(0, t)+u^{2}(0, t)\right]=\dot{C}(t) .
\end{aligned}
$$

Comparing (6c) with (5) and making use of (6b) with (2b), we can restate the compatibility condition for $C(t)$, (5), in the following shape:

$$
\dot{C}(t)=C(t) F(t)+\frac{v^{2}(0, t)}{C(t)} .
$$

According to $(6 c)$, the boundary datum $v_{x}(0, t)$ for the heat equation (4) is a nonlinear combination of known $\left(u_{x}(0, t)\right)$ and unknown $(u(0, t))$ boundary data for the Burgers equation (1).

The Neumann problem on the semi-line for $\mathrm{v}(x, t)$ is then in principle solved through the following prescription:

1. Solve the Neumann problem on the semi-line for $v(x, t)$, with initial datum (6a) and $(6 \mathrm{c})$;

2. Determine the unknown function $C(t)$ by means of the transformation (3a) and (3c);

3. Recover $u(x, t)$ via the inverse transformation (3b).

We reduced the problem of solving Burgers equation (1) with the Neumann condition $(2 \mathrm{a}-2 \mathrm{e})$ to the determination of the couple $\{v(x, t), C(t)\}$, where $\mathrm{v}(x, t)$ solves the heat equation (4) with the Neumann condition (6a-6c) and $C(t)$ simultaneously satisfying (7).

In order to explicitly evaluate the solution $\mathrm{v}(x, t)$ of $(4)$, as in [12], it is convenient to introduce the cosine-Fourier transform:

$$
\begin{aligned}
& v(x, t)=\frac{2}{\pi} \int_{0}^{\infty} \mathrm{d} k \hat{v}(k, t) \cos (k, x), \\
& \hat{v}(k, t)=\int_{0}^{\infty} \mathrm{d} x v(x, t) \cos (k, x) \\
& =\hat{v}(k, 0) \mathrm{e}^{-k^{2} t}-\int_{0}^{t} \mathrm{~d} \tilde{t} v_{x}(0, \tilde{t}) \mathrm{e}^{-k^{2}(\tilde{t}-t)} \\
& =\hat{v}(k, 0) \mathrm{e}^{-k^{2} t}-\int_{0}^{t} \mathrm{~d} \tilde{t} \dot{C}(\tilde{t}) \mathrm{e}^{-k^{2}(\tilde{t}-t)}, \\
& \hat{v}(k, 0)=\int_{0}^{\infty} \mathrm{d} x v(x, 0) \cos (k x) \\
& =\int_{0}^{\infty} \mathrm{d} x u_{0}(x) \cos (k, x) e^{\int_{0}^{x} \mathrm{~d} \tilde{x} u_{0}(\tilde{x})} .
\end{aligned}
$$


Using (8b) and (8c) with (8a), via (7), we equivalently get

$$
\begin{aligned}
& v(x, t)=\frac{2}{\pi} \int_{0}^{\infty} \mathrm{d} k \hat{v}(k, 0) \cos (k x) \mathrm{e}^{-k^{2} t} \\
& -\frac{2}{\pi} \int_{0}^{\infty} \mathrm{d} k \int_{0}^{t} \mathrm{~d} \tilde{t}\left[C(\tilde{t}) F(\tilde{t})+\frac{v^{2}(0, \tilde{t})}{C(\tilde{t})}\right] \cos (k x) \mathrm{e}^{k^{2}(\tilde{t}-t)} .
\end{aligned}
$$

In the right-hand side of (9), $\mathrm{v}(0, t)$ is unknown. It is then convenient to set $z(t)=$ $v(0, t)$ and to calculate left- and right-hand sides of (9) at $x=0$. To do so, let us start by recalling that

(a) for an arbitrary function of $t, g(t)$, we have

$$
\int_{0}^{\infty} \mathrm{d} k \int_{0}^{t} \mathrm{~d} \tilde{t} g(\tilde{t}) \mathrm{e}^{k^{2}(\tilde{t}-t)}=\frac{\sqrt{\pi}}{2} \int_{0}^{t} \mathrm{~d} \tilde{t} \frac{g(\tilde{t})}{\sqrt{t-\tilde{t}}} ;
$$

b) from the convolution properties of the cosine-Fourier transform (8), we have

$$
\frac{2}{\pi} \int_{0}^{\infty} \mathrm{d} k \hat{v}(k, 0) \mathrm{e}^{-k^{2} t}=\frac{1}{\sqrt{\pi t}} \int_{0}^{\infty} \mathrm{d} x v(x, 0) \mathrm{e}^{-\frac{x^{2}}{4 t}} .
$$

From this, at $x=0$, we can restate (9) as follows:

$$
z(t)=\frac{1}{\sqrt{\pi t}} \int_{0}^{\infty} \mathrm{d} x v(x, 0) \mathrm{e}^{-\frac{x^{2}}{4 t}}-\frac{1}{\sqrt{\pi}} \int_{0}^{t} \mathrm{~d} \tilde{t} \frac{\dot{C}(\tilde{t})}{\sqrt{t-\tilde{t}}}
$$

or, via (7),

$$
\begin{aligned}
& z(t)=\frac{1}{\sqrt{\pi t}} \int_{0}^{\infty} \mathrm{d} x v(x, 0) \mathrm{e}^{-\frac{x^{2}}{4 t}}-\frac{1}{\sqrt{\pi}} \int_{0}^{t} \mathrm{~d} \tilde{t} \frac{1}{\sqrt{t-\tilde{t}}}\left(C(\tilde{t}) F(\tilde{t})+\frac{z^{2}(\tilde{t})}{C(\tilde{t})}\right) . \\
& \dot{C}(t)=C(t) F(t)+\frac{z^{2}(t)}{C(t)}, \quad C(0)=1 .
\end{aligned}
$$

Making use of (10a), we can put in a more explicit form the previously given prescription to solve the Neumann problem on the semi-line, (1) and (2a-2e), for $u(x, t)$ :

1. Given the Neumann data on the semi-line, $(2 \mathrm{a}-2 \mathrm{e})$, compute $C(t)$ by substituting (10b) into (10c), namely, from the following nonlinear integro-differential equation:

$$
\begin{aligned}
& C(t) \dot{C}(t)=F(t) C^{2}(t) \\
& +\left(\frac{1}{\sqrt{\pi t}} \int_{0}^{\infty} \mathrm{d} x u_{0}(x) \mathrm{e}^{-\frac{x^{2}}{4 t} \int_{0}^{x} \mathrm{~d} \tilde{x} u_{0}(\tilde{x})}-\frac{1}{\sqrt{\pi}} \int_{0}^{t} \mathrm{~d} \tilde{t} \frac{\dot{C}(\tilde{t})}{\sqrt{t-\tilde{t}}}\right)^{2}
\end{aligned}
$$

with $C(0)=1$ as in $(3 \mathrm{c})$;

2. Evaluate the solution to the heat equation (4) with IBV data $(6 a-6 c), v(x, t)$, by means of (8a) making use of (8b) and (8c);

3. Recover $u(x, t)$ from $v(x, t)$ via (3b).

For arbitrary $u_{0}(x)$ and $F(t)$, there is no general technique for solving a nonlinear integro-differential equation like (11). On the other hand, the determination of the solution $u(x, t)$ has been reduced to the solution of the nonlinear integral equation (10b)-with $C(t)$ satisfying (10c)-for only one independent variable $(t)$. In the next section, we prove the existence and uniqueness of the function $z(t)$ for $0 \leq t<\sigma$, with 0 
$<\sigma<\infty$ (Lemma 1). Once the existence and uniqueness of $z(t)$ are established, the existence and uniqueness of $\mathrm{v}(x, t)$ for $0 \leq t<\sigma$ then follow, via (9), with $C(t)$ being obtained via (10c). Then, via the inverse transformation (3b), Theorem 1 immediately follows, namely the solution of the original Neumann problem (2a-2e) for the Burgers equation (1) exists and is unique (for $0 \leq t<\sigma$ ).

\section{Contraction mapping}

In order to analyze the existence properties of $z(t)$ for $0 \leq t<\sigma<\infty$, we denote by $S_{M}$ $(\sigma)$ the closed sphere $\|z\| \leq M$ in the Banach space of continuous functions $z(t)$ for $t$ $\in[0, \sigma)$, with the uniform norm ||$z \|=l . u \cdot b \cdot|z(t)|$. On the sphere $S_{M}(\sigma)$, we introduce the transformation:

$$
w(t)=\mathcal{T} z(t),
$$

where $\mathcal{T} z(t)$ coincides with the right-hand side of (10b). To prove the existence and the uniqueness of the solution of the integral equation (10b) for a finite interval of time, we will prove the following:

Lemma 1 The mapping operator $\mathcal{T}$ is a contraction mapping in $S_{M}(\sigma)$ for $t \in[0, \sigma)$.

In order to prove this Lemma, we need to prove that, for $t \in[0, \sigma), \mathcal{T}$ is closed and contractive in $S_{M}(\sigma)$.

\subsection{Closure of $\mathcal{T}$ in $S_{M}(\sigma)$ for $t \in[0, \sigma)$}

We need to prove that if $z(t) \in S_{M}(\sigma)$ then $w(t) \in S_{M}(\sigma)$ as well, namely that $\|z(t)\| \leq$ $M$ for $t \in[0, \sigma)$ entails $\|w(t)\|=\|\mathcal{T} z(t)\| \leq M$ for $t \in[0, \sigma)$.

The first step is to obtain an upper and lower bounds for $|C(t)|$. Integrating (10c), we obtain

$$
C^{2}(t)=\mathrm{e}^{2 \int_{0}^{t} \mathrm{~d} t^{\prime} F\left(t^{\prime}\right)}\left[1+2 \int_{0}^{t} \mathrm{~d} t^{\prime} z^{2}(t) \mathrm{e}^{-2 \int_{0}^{t^{\prime}} \mathrm{d} t^{\prime \prime} F\left(t^{\prime \prime}\right)}\right] .
$$

From the fact that $\left|\mathrm{e}^{x}\right|=\mathrm{e}^{x} \leq \mathrm{e}^{|x|}$ for any $x \in \mathbb{R}$, applying the triangular inequality $(|x|$ $-|y| \leq|x+y|<|x|+|y|)$ on (13) we get

$$
\begin{aligned}
|C(t)| & \leq\left|\mathrm{e}^{\int_{0}^{t} \mathrm{~d} t^{\prime} F\left(t^{\prime}\right)}\right|\left[1+2\left|\int_{0}^{t} \mathrm{~d} t^{\prime} z^{2}(t) \mathrm{e}^{-2 \int_{0}^{t^{\prime}} \mathrm{d} t^{\prime \prime} F\left(t^{\prime \prime}\right)}\right|\right]^{1 / 2} \\
& \leq \mathrm{e}^{\sigma B}\left[1+2 M^{2} \sigma \mathrm{e}^{2 \sigma B}\right]^{1 / 2}, \\
|C(t)| & \geq\left|\mathrm{e}^{\int_{0}^{t} \mathrm{~d} t^{\prime} F\left(t^{\prime}\right)}\right|\left[1-2\left|\int_{0}^{t} \mathrm{~d} t^{\prime} z^{2}(t) \mathrm{e}^{-2 \int_{0}^{t^{\prime}} \mathrm{d} t^{\prime \prime} F\left(t^{\prime \prime}\right)}\right|\right]^{1 / 2} \\
& \leq \mathrm{e}^{\sigma B}\left[1+2 M^{2} \sigma \mathrm{e}^{2 \sigma B}\right]^{1 / 2} ;
\end{aligned}
$$

imposing the last right-hand side of (14b) to be strictly greater than zero, we have the following condition on $\sigma$ :

$$
0 \leq \sigma<\frac{W\left(\frac{B}{M^{2}}\right)}{2 B}
$$

where $W$ is the Lambert- $W$ function, implicitly defined as the inverse function of $f$ $(W)=W \mathrm{e}^{W}$ 
The second step, is to obtain an upper bound for $\mathcal{T} z(t)$. Applying the triangular inequality on (10b), via (16), we get

$$
\begin{array}{r}
\|\mathcal{T} z(t)\| \leq\left|\frac{1}{\sqrt{\pi t}}\right| \int_{0}^{\infty} \mathrm{d} x v(x, 0) \mathrm{e}^{-\frac{x^{2}}{4 t}} \\
+\frac{1}{\sqrt{\pi}}\left|\int_{0}^{t} \mathrm{~d} \tilde{t} \frac{C(\tilde{t}) F(\tilde{t})}{\sqrt{t-\tilde{t}}}\right| \\
+\frac{1}{\sqrt{\pi}}\left|\int_{0}^{t} \mathrm{~d} \tilde{t} \frac{z^{2}(\tilde{t})}{C(\tilde{t}) \sqrt{t-\tilde{t}}}\right|
\end{array}
$$

From (6a) and (2e) we can write

$$
v(0, x) \leq\left\|u_{0}(x)\right\| \exp \left(\left\|u_{0}(x)\right\| \equiv A\right) ;
$$

then, for the first term on the right-hand side of (15), we get

$$
\left|\frac{1}{\sqrt{\pi t}} \int_{0}^{\infty} \mathrm{d} x v(x, 0) \mathrm{e}^{-\frac{x^{2}}{4 t}}\right| \leq A\left|\frac{1}{\sqrt{\pi t}} \int_{0}^{\infty} \mathrm{d} x \mathrm{e}^{-\frac{x^{2}}{4 t}}\right|=A .
$$

For the second and the third terms in the right-hand side of (15), inequalities (14a$14 \mathrm{c})$ and $(2 \mathrm{~d})$ entail

$$
\begin{aligned}
& \frac{1}{\sqrt{\pi}}\left|\int_{0}^{t} \mathrm{~d} \tilde{t} \frac{C(\tilde{t}) F(\tilde{t})}{\sqrt{t-\tilde{t}}}\right| \leq \frac{2 B \sqrt{\sigma} \mathrm{e}^{\sigma B}}{\sqrt{\pi}} \sqrt{1+2 M^{2} \sigma \mathrm{e}^{2 \sigma B}} \\
& \frac{1}{\sqrt{\pi}}\left|\int_{0}^{t} \mathrm{~d} \tilde{t} \frac{z^{2}(\tilde{t})}{C(\tilde{t}) \sqrt{t-\tilde{t}}}\right| \leq \frac{2 M^{2} \sqrt{\sigma}}{\sqrt{\pi} \mathrm{e}^{\sigma B} \sqrt{1-2 M^{2} \sigma \mathrm{e}^{2 \sigma B}}} .
\end{aligned}
$$

Defining $\mathrm{M}=\alpha A$, with $\alpha>1$, and combining (15) with (17a-17c), we get

$$
\|w(t)\| \leq M\left[\frac{1}{\alpha}+\beta(\sigma)\right]
$$

where

$$
\beta(\sigma)=\frac{2 \sqrt{\sigma}}{\sqrt{\pi}}\left(B \mathrm{e}^{\sigma B} \sqrt{\frac{1}{M^{2}}+2 \sigma \mathrm{e}^{2 \sigma B}}+\frac{1}{\mathrm{e}^{\sigma B} \sqrt{\frac{1}{M^{2}}-2 \sigma \mathrm{e}^{2 \sigma B}}}\right),
$$

with $\sigma$ satisfying condition (14c). On the other hand, in the interval $\sigma \in\left[0, \frac{w\left(B / M^{2}\right)}{2 B}\right]$, for $B>0$ and $M>0, \beta(\sigma)$ is a monotonic, increasing bijective function on the positive Reals, and so there exists a value $\sigma *<\frac{w\left(B / M^{2}\right)}{2 B}$ such that

$$
\frac{1}{\alpha}+\beta(\sigma) \leq 1 \quad \forall \quad \sigma \in[0, \sigma *]
$$

Taking $\sigma \leq \sigma^{*}$, from (18a), we have $\|w(t)\| \leq M$. Thus the mapping $\mathcal{T}$ is closed. 
3.2 Contractivity of $\mathcal{T}$ in $S_{M}(\sigma)$ for $t \in[0, \sigma)$

We need to prove that, given two solutions of (12), $z(t)$ and $\hat{z}(t)$, with $\|z(t)-\hat{z}(t)\|=\delta<$ $2 M$; it then follows that $\|\mathcal{T} z(t)-\mathcal{T} \hat{z}(t)\| \leq \theta \delta$ with $0<\theta<1$.

We now write

$$
\begin{aligned}
\|w-\hat{w}\| & =\frac{1}{\sqrt{\pi}}\left|\int_{0}^{t} \mathrm{~d} \tilde{t} \frac{F(\tilde{t})[C(\tilde{t})-\hat{C}(\tilde{t})]}{\sqrt{t-\tilde{t}}}\right|+\int_{0}^{t} \mathrm{~d} \tilde{t} \frac{1}{\sqrt{t-\tilde{t}}}\left[\frac{z^{2}(\tilde{t})}{C(\tilde{t})}-\frac{\hat{z}^{2}(\tilde{t})}{\hat{C}(\tilde{t})}\right] \\
& =\frac{1}{\sqrt{\pi}}\left|\int_{0}^{t} \mathrm{~d} \tilde{t} \frac{\dot{C}(\tilde{t})-\dot{\hat{C}}(\tilde{t})}{\sqrt{t-\tilde{t}}}\right| .
\end{aligned}
$$

Let us recall

$$
X(t)=\left|\frac{z(t)}{C(t)}\right| \quad \text { and } \quad \hat{X}(t)=\left|\frac{\hat{z}(t)}{\hat{C}(t)}\right| .
$$

Notice that, if $\sigma \leq \sigma *<\left[0, \frac{w\left(B / M^{2}\right)}{2 B}\right]$, then, for $0 \leq t \leq \sigma$, via (14a) and (14b), we have that $C(t)$ is a nonzero bounded function of $t$ :

$$
e^{\sigma B}\left[1-2 M^{2} \sigma e^{2 \sigma B}\right]^{1 / 2} \leq|C(t)| \leq e^{\sigma B}\left[1+2 M^{2} \sigma e^{2 \sigma B}\right]^{1 / 2}
$$

thus $X(t)$ and $\hat{X}(t)$ are bounded functions of $t$ as well.

The identity

$$
\left[\frac{z^{2}(t)}{C(t)}-\frac{\hat{z}^{2}(t)}{\hat{C}(t)}\right]=[z(t)-\hat{z}(t)]\left[\frac{z(t)}{C(t)}+\frac{\hat{z}(t)}{\hat{C}(t)}\right]-\frac{z(t)}{C(t)} * \frac{\hat{z}(t)}{\hat{C}(t)}[C(t)-\hat{C}(t)],
$$

entails

$$
\begin{aligned}
|\dot{C}(t)-\dot{\hat{C}}(t)| & \leq[B+X(t) \hat{X}(t)]|C(t)-\hat{C}(t)|+|z(t)-\hat{z}(t)|[X(t)+\hat{X}(t)] \\
& =\left[B+\tilde{R}_{1}(t)\right]|C(t)-\hat{C}(t)|+\tilde{R}_{2}(t)|z(t)-\hat{z}(t)|,
\end{aligned}
$$

where

$$
\tilde{R}_{1}(t)=X(t) \hat{X}(t) \quad \text { and } \quad \tilde{R}_{2}(t)=X(t) \hat{X}(t) .
$$

Let us recall

$$
R_{1}=\max _{0 \leq t \leq \sigma *}\left\{\tilde{R}_{1}(t)\right\} \text { and } R_{2}=\max _{0 \leq t \leq \sigma *}\left\{\tilde{R}_{2}(t)\right\},
$$

existence and well-definedness of which are implied by our previous considerations about the boundedness of $C(t)$.

Since, for an arbitrary function of $t, g(t)$, we have that $\left|\frac{\mathrm{d} g(t)}{\mathrm{d} t}\right| \geq\left|\frac{\mathrm{d}|g(t)|}{\mathrm{d} t}\right|$, from (22), we can write

$$
\left|\frac{\mathrm{d}|C(t)-\hat{C}(t)|}{\mathrm{d} t}\right| \leq\left(B+R_{1}\right)|C(t)-\hat{C}(t)|+\delta R_{2},
$$


or, integrating once with initial condition $|C(0)-\hat{C}(0)|=0$,

$$
\begin{aligned}
& |C(t)-\hat{C}(t)| \leq\left[\mathrm{e}^{\left(B+R_{1}\right)_{\sigma}}-1\right] \frac{\delta R_{2}}{B+R_{1}}, \\
& \left|\frac{\mathrm{d}|C(t)-\hat{C}(t)|}{\mathrm{d} t}\right| \leq \delta R_{2} \mathrm{e}^{\left(B+R_{1}\right) \sigma} .
\end{aligned}
$$

Formula (25b), via (19), implies

$$
\|w(t)-\hat{w}(t)\| \leq \theta \delta \quad \text { with } \quad \theta=\frac{2 \sqrt{\sigma} R_{2}}{\sqrt{\pi}} \mathrm{e}^{\left(B+R_{1}\right) \sigma} .
$$

Choosing

$$
\sigma<\min \left\{\sigma *, \frac{W\left(\frac{\pi\left(2+R_{1}\right)}{2 R_{2}^{2}}\right)}{2\left(2+R_{1}\right)}\right\}
$$

we get $\theta<1$ and, for what we saw in the previous Subsection 3.1, the mapping $\mathcal{T}$ remains closed. Thus, Lemma 1 is proved, namely, $\mathcal{T}$ is a contraction operator on $S_{M}$ $(\sigma)$.

Lemma 1 means that there exists a unique fixed point $z(t)=\mathcal{T} z(t)$ of $\mathcal{T}$ in $S_{M}(\sigma)$, for $0 \leq t \leq \sigma$. We have thereby proven the existence and uniqueness of the solution of the integral equation (10b) for $0 \leq t<\sigma$. Then, as explained at the end of Section 2, from the existence and uniqueness of $z(t)$ in the interval $0 \leq t<\sigma$, we get, via (9) and (10c), the existence and uniqueness of $\mathrm{v}(x, t)$ in the same interval, and via the inverse transformation (3b), we immediately get Theorem 1 .

\section{A special solution}

In this section, we consider a particular solution of the Neumann problem (2a-2e) for the Burgers equation (1), and derive the corresponding expression for $z(t)$.

A solution to the Burgers equation is given by

$$
u(x, t)=\frac{\left(1-\mathrm{e}^{A}\right) \mathrm{e}^{-\frac{x^{2}}{4\left(t+t_{0}\right)}}}{\sqrt{\pi\left(t+t_{0}\right)}\left\{2-\left(1-\mathrm{e}^{A}\right)\left[1-\operatorname{erf}\left(\frac{x}{2\left(t+t_{0}\right)}\right)\right]\right\}},
$$

where $A$ and $t_{0}>0$ are two real constants and

$$
\operatorname{erf}(x)=\frac{2}{\sqrt{\pi}} \int_{0}^{x} \mathrm{~d} \xi \mathrm{e}^{-\xi^{2}} .
$$

From (28), we obtain for the squared modulus

$$
|u(x, t)|^{2}=\frac{H^{2}[y(x, t)]}{t+t_{0}},
$$

Where

$$
H(y)=\left\{\sqrt{\pi} \mathrm{e}^{y^{2}}\left[\operatorname{coth}\left(\frac{A}{2}\right)-\operatorname{erf}(y)\right]\right\}^{-1},
$$


and

$$
y(x, t)=\frac{x}{2 \sqrt{t+t_{0}}} .
$$

Thus the solution (28) on the whole line is a single hump with (negative) peak value given by

$$
u\left(x_{p}, t\right)=\frac{y_{p}}{\sqrt{t+t_{0}}},
$$

where $y_{p}$ is the solution of the equation

$$
y_{p}=H\left(y_{p}\right)
$$

the peak value is attained at

$$
x_{p}=2 y_{p} \sqrt{t+t_{0}}
$$

and moves to the left with velocity

$$
\frac{\mathrm{d} x_{p}}{\mathrm{~d} t}=\frac{y_{p}}{\sqrt{t}} .
$$

The corresponding initial datum and boundary condition, which associate the given solution (28) to the Burgers equation (1) are

$$
\begin{aligned}
& u(x, 0)=u_{0}(x)=\frac{\left(1-\mathrm{e}^{A}\right) e^{-\frac{x 2}{4 t_{0}}}}{\sqrt{\pi t_{0}}\left\{2-\left(1-\mathrm{e}^{A}\right)\left[1-\operatorname{erf}\left(\frac{x}{2 t_{0}}\right)\right]\right\}}, \\
& u_{x}(0, t)=F(t)=-\frac{\tanh ^{2}\left(\frac{A}{2}\right)}{\pi\left(t+t_{0}\right)} .
\end{aligned}
$$

Notice that, if $t_{0}=0$, then, from (31a), it turns out that $u_{0}(x)=A \delta(x)$, where $\delta(x)$ is the Dirac delta function; in this case, all the following calculations can still be performed.

Next, we prove that (28) considered on the semi-line $x \in[0,+\infty)$ is a particular solution of the Neumann problem (2a-2e) for the Burgers equation (1). To this end, we start noting that from the solution (28), we get

$$
u(0, t)=-\frac{\tanh \left(\frac{A}{2}\right)}{\sqrt{\pi\left(t+t_{0}\right)}},
$$

so that

$$
u_{x}(0, t)+u^{2}(0, t)=0 ;
$$

this last relation, via (5) and (3c), implies

$$
C(t)=1,
$$

which in turn, from (6b) and (32), implies

$$
z(t)=v(0, t)=u(0, t)=-\frac{\tanh \left(\frac{A}{2}\right)}{\sqrt{\pi\left(t+t_{0}\right)}} .
$$


On the other hand, it is now immediate to see that the integral equation (10b), when (34) and (35) are used, reduces to

$$
\begin{aligned}
& z(t)=\frac{1}{\sqrt{\pi t}} \int_{0}^{\infty} \mathrm{d} x v(x, 0) \mathrm{e}^{-\frac{x^{2}}{4 t}}, \\
& v(x, 0)=-\frac{\tanh \left(\frac{A}{2}\right)}{\sqrt{\pi t_{0}}} \mathrm{e}^{-\frac{x^{2}}{4 t_{0}}} .
\end{aligned}
$$

An explicit computation of the integral (36a) with (36b) yields immediately the same expression (35) for $z(t)$.

\section{Acknowledgements}

The authors wish to thank M. J. Ablowitz for enlightening discussions on this topic. MS wishes to thank T. Horikis and M. Hoefer, particularly the latter for bringing to his attention a different way to linearize (1).

\section{Author details}

${ }^{1}$ Dipartimento di Matematica ed Informatica, Università degli Studi di Perugia, 06123 Perugia, Italy ${ }^{2}$ INFN, Istituto Nazionale di Fisica Nucleare, Sezione di Perugia, Italy ${ }^{3}$ Institut des Hautes Etudes Scientifiques, 91440 Bures-sur-Yvette, France

\section{Authors' contributions}

The authors declare that the work was realized in collaboration with the same responsibility. All authors read and approved the final manuscript.

\section{Competing interests}

The authors declare that they have no competing interests.

Received: 28 April 2011 Accepted: 14 October 2011 Published: 14 October 2011

\section{References}

1. Fokas, AS, Its, AR: Soliton generation for initial-boundary value problems. Phys Rev Lett. 68, 3117-3120 (1992)

2. Fokas, AS: A unified transform method for solving linear and certain nonlinear PDEs. Proc R Soc Lond A. 53, 1411-1443 (1997)

3. Degasperis, A, Manakov, SV, Santini, PM: On the initial-boundary value problem for soliton equations. JEPT Lett. 74 , 481-485 (2001)

4. Degasperis, A, Manakov, SV, Santini, PM: Initial-boundary value problems for linear and soliton PDEs. Theor Math Phys. 133, 1475-1489 (2002)

5. Fokas, AS: On the integrability of linear and nonlinear partial differential equations. J Math Phys. 41, 4188-4237 (2000)

6. Fokas, AS, Pelloni, P: Two-point boundary value problems for linear evolution equations. Math Proc Camb Phil Soc. 131, 521-543 (2001)

7. Grinevich, P, Santini, PM: The initial-boundary value problem on the interval for the nonlinear Schrdinger equation. The algebro-geometric approach. I Am Math Soc Transl 212, 157-178 (2004). Adv. Math. Sci. Appl. 55

8. Fokas, AS, Stuart, JT: The time periodic solution of the Burgers equation on the half-line and an application to steady streaming. J Nonlinear Math Phys. 12, 302-314 (2005)

9. Calogero, F, De Lillo, S: The Eckhaus PDE $i \psi_{t}+\psi_{x x}+2\left(|\psi|^{2}\right)_{x} \psi+|\psi|^{4} \psi=0$. Inverse Problems. 3, $633-681$ (1987)

10. Calogero, F, De Lillo, S: Cauchy problems on the semiline and on a finite interval for the Eckhaus equation. Inverse Problems. 4, L33-L37 (1988)

11. Calogero, F, De Lillo, S: The Burgers equation on the semi-infinite and finite intervals. Nonlinearity. 2, 27-43 (1989)

12. Calogero, F, De Lillo, S: Burgers equation on the semiline. Inverse Problems. 5, L37-L40 (1989)

13. Calogero, F, De Lillo, S: The Burgers equation on the semiline with general boundary conditions at the origin. J Math Phys. 32, 99-105 (1991)

14. Ablowitz, MJ, De Lillo, S: Forced and semiline solutions of the Burgers equation. Phys Lett A. 156, $483-487$ (1991)

15. De Lillo, S: Neumann problem on the semi-line for the Eckhaus equation. Nonlinearity. 18, 2365-2372 (2005)

16. Zhu, M, Zhao, Z: Optimal Control of Nonlinear Strength Burgers Equation under the Neumann Boundary Condition. Int J Nonlinear Sci. 2(1), 111-118 (2006)

doi:10.1186/1687-2770-2011-34

Cite this article as: De Lillo and Sommacal: Neumann problem on the semi-line for the Burgers equation. Boundary Value Problems 2011 2011:34. 